\title{
Noninvasive Diagnosis of Liver Fibrosis and Cirrhosis in Chronic Hepatitis C Patients
}

\author{
Abdelfattah M. Attallah, ${ }^{1 *}$ Mohamed El-Far, ${ }^{2}$ Mohamed M. Omran, ${ }^{3}$ Khaled Farid, ${ }^{4}$ \\ Mohamed S. Albannan, ${ }^{1}$ and Ibrahim El-Dosoky ${ }^{5}$ \\ ${ }^{1}$ Research \& Development Department, Biotechnology Research Center (BRC), New Damietta City, Egypt \\ ${ }^{2}$ Faculty of Science, Mansoura University, Mansoura, Egypt \\ ${ }^{3}$ Faculty of Science, Helwan University, Cairo, Egypt \\ ${ }^{4}$ Tropical Medicine department, Faculty of Medicine, Mansoura University, Mansoura, Egypt \\ ${ }^{5}$ Pathology Department, Faculty of Medicine, Mansoura University, Mansoura, Egypt
}

\begin{abstract}
Background: We aimed to derive a simple noninvasive test for liver-fibrosis staging and then estimate its performance against four simple noninvasive tests in chronic hepatitis $\mathrm{C}(\mathrm{CHC})$ patients. Methods: $\mathrm{CHC}$ patients were divided into two cohorts: an estimation set $(n=324)$ and a validation set $(n=524)$. Liver fibrosis was staged according to the METAVIR scoring system. Statistical analysis was done using stepwise linear discriminant analysis and area under receiver-operating characteristic curves (AUCs). Results: Biotechnology Research Center (BRC) score was constructed combining several blood markers that proved useful to stage liver fibrosis. Aspartate aminotransferase /alanine aminotransferase ratio (AAR), aspartate to platelet
\end{abstract}

Key words: Hepatitis C virus; blood markers; Biotechnology Research Center score; liver biopsy; APRI ratio index (APRI), Fibro- $\alpha$, King, and BRC scores correlated with the histological fibrosis stages with correlation coefficient 0.26 , $0.36,0.58,0.45$, and 0.73 , respectively. BRC score produced AUCs $0.87,0.83$, and 0.89 for significant (F2-F4), advanced fibrosis (F3-F4), and cirrhosis (F4), respectively. These results were reproduced in the validation study with no significant difference yielding AUCs 0.85 for F2-F4, 0.82 for F3-F4, and 0.88 for F4. Conclusion: BRC score, a novel noninvasive test, is a useful and easy tool to evaluate liver fibrosis in $\mathrm{CHC}$ patients and seems more efficient than AAR, APRI, Fibro- $\alpha$ score, and King's score in this group of Egyptian patients. J. Clin. Lab. Anal. 27:121-129,

2013. (c) 2013 Wiley Periodicals, Inc.
Biotechnology Research Center score; liver

\section{INTRODUCTION}

Approximately 170 million people worldwide are chronically infected with hepatitis $\mathrm{C}$ virus (HCV), but most are unaware of the infection (1). The global seroprevalence of anti-HCV antibodies is estimated to be $3 \%$, with a higher prevalence in countries in the Mediterranean Basin, Far East, Africa, and Central America (2). Strikingly, 14\% of the general population in Egypt is infected with $\mathrm{HCV}$ (3). Approximately $90 \%$ of Egyptian HCV isolates belong to a single subtype, $4 \mathrm{a}$ (4). Liver fibrosis is the final common pathway of most chronic liver diseases (8) of various etiologies such as hepatitis B, C and nonalcoholic steatohepatitis (5). The stage of fibrosis is prognostic and provides information on the likelihood of disease progression and response to treatment $(6,7)$. The assessment of the stage of liver fibrosis is essential for prognosis and for deciding on antiviral treatment (8). Currently, liver biopsy is the gold standard for staging liver fibrosis. However, the accuracy of liver biopsy to assess fibrosis has also been questioned, in relation to sampling errors and intra- and interobserver variability that may lead to over- or under-staging. The size of the biopsy specimen

\footnotetext{
* Correspondence to: Professor of immunology and genetics. Abdelfattah M. Attallah, Biotechnology Research Center, P.O. Box 14, 23 July St., Industrial Zone, New Damietta 34517, Egypt. E-mail: amattallah@hotmail.com

Received 10 September 2012; Accepted 14 December 2012 DOI 10.1002/jcla.21572

Published online in Wiley Online Library (wileyonlinelibrary.com).
} 
represents $1 / 50,000$ of the total mass of the liver and thus carries substantial sampling error. For instance, it has been shown that only $65 \%$ of $15-\mathrm{mm}$ biopsies and $75 \%$ of 25-mm biopsies were correctly staged (9). Also, a difference of at least one fibrosis stage between the right and left lobes has been reported in around $30 \%$ of cases (10). In addition, liver biopsy is associated with life-threatening complications and pain $(11,12)$. These limitations have led to the development of noninvasive methodologies for the assessment of fibrosis. While several noninvasive models utilize tests that are not routinely available and are of additional costs, the so-called "direct markers," others incorporate routine clinical and laboratory data but do not directly reflect extracellular matrix metabolism, the socalled "indirect markers" $(13,14)$. But the cost and complexity of these direct markers greatly reduce their clinical usefulness. Ideally, noninvasive alternatives should be simple, cheap, easy to perform, safe, precise, reproducible, and capable of differentiating patients in need of therapy $(15,16)$. Several indices or predictors of hepatic fibrosis have been proposed including aspartate aminotransferase (AST)/alanine aminotransferase (ALT) ratio (AAR) (17-19), aspartate to platelet ratio index (APRI) (20), Fibro- $\alpha$ score (21), and King's score (22). The aim of this study was to derive a simple noninvasive test for liverfibrosis staging and then estimate its performance against that of the other simple noninvasive tests in chronic hepatitis $\mathrm{C}(\mathrm{CHC})$.

\section{PATIENTS AND METHODS}

\section{Patients}

A total of 324 consecutive Egyptian patients with clinically and laboratory confirmed CHC constituted the estimation group whereas 524 patients with $\mathrm{CHC}$ constituted the validation group. All tissue and serum samples were obtained with informed consent. All patients were negative for other causes of chronic liver disease including viral hepatitis A and B. Exclusion criteria for the study were age greater than 65 years, a history of habitual alcohol consumption or hepatocellular carcinoma, previous interferon treatment, decompensated liver disease (ascites, jaundice, variceal bleeding, or encephalopathy), evidence of coexistent liver disease, and liver transplantation. All patients were tested positive for the presence of HCVRNA using quantitative polymerase chain reaction assay (COBAS Ampliprep/ COBAS TaqMan, Roche Diagnostics, Pleasanton).

\section{Blood Samples and Liver Biopsies}

Blood samples were collected by vein-puncture within 2 weeks of liver biopsy. Complete blood count was per- formed on blood treated with EDTA-K3 using KX-21 Sysmex automated hematology analyzer (Sysmex Corporation, Japan), and another portion was treated with a citrate solution for prothrombin-INR (international normalized ratio, INR). Liver function tests (albumin, total bilirubin, AST, ALT, and alkaline phosphatase (ALP)) were all measured on fresh serum on an automated biochemistry analyzer (Roche/Hitachi 917 Multichannel Analyzer). Alpha fetoprotein (AFP) level was estimated by chemiluminescence, with Immulite (1000) AFP kit (Diagnostic Products Corporation; Los Angeles, CA, USA). AAR (17-19) was calculated as AST/ALT. APRI (20) was calculated as [(AST/upper limit of normal) / platelet count $\left.\left(10^{9} / 1\right)\right] \times 100$. Fibro- $\alpha$ score $(21)$ was calculated as $1.35+\mathrm{AFP}(\mathrm{U} / 1) \times 0.009584+\mathrm{AAR} \times 0.243$-platelet count $\left(10^{9} / 1\right) \times 0.001624$. King's score $(22)$ was calculated as age $\times \operatorname{AST}(\mathrm{U} / 1) \times \mathrm{INR} /$ platelet count $\left(10^{9} / 1\right)$. Needle liver biopsy specimens were obtained with an 18gauge or larger needle. To be considered as adequate for scoring, the liver biopsies had to measure at least $15 \mathrm{~mm}$ and/or contain at least five portal tracts, except for cirrhosis for which no limitation was required. Biopsies were interpreted according to METAVIR scoring system (23). Liver pathologists under one of the author's supervision were blinded to patient clinical and laboratory characteristics. Fibrosis was scored on a 5-point scale: F0, no fibrosis; F1, portal fibrosis alone; F2, portal fibrosis with rare septae; F3, portal fibrosis with many septae; F4, cirrhosis. Significant fibrosis was defined as a METAVIR score of $>1$ (F2-F4), advanced fibrosis was defined as a METAVIR score of $>2$ (F3-F4), cirrhosis was defined as a METAVIR score of $>3$ (F4).

\section{Statistical Analysis}

All statistical analyses were performed by SPSS software version 15.0 (SPSS Inc., Chicago, IL). Continuous variables were expressed as mean \pm standard deviation. A value of $P<0.05$ was considered statistically significant. The correlation was evaluated by Spearman's rank correlation coefficient. Patients were divided into three main groups as patients with significant fibrosis, patients with advanced fibrosis, and patients with cirrhosis. The main endpoint was the identification of patients with clinically significant fibrosis (F2-F4) vs. those without (F0-F1) using a simple score based on routine laboratory markers.

Significant fibrosis has been adopted as a target because individuals with significant fibrosis are at increased risk of developing cirrhosis and are usually treated (24). For formulation of the predictive score, univariate analysis was performed to identify variables that were significantly different between patients with clinically significant fibrosis vs. those without it. All variables with a high area under receiver-operating characteristic curve (AUC) and 
TABLE 1. Comparison of Patients Characteristics in the Estimation and Validation Groups

\begin{tabular}{|c|c|c|c|}
\hline Marker & $\begin{array}{c}\text { Estimation group } \\
\quad(n=324)\end{array}$ & $\begin{array}{l}\text { Validation group } \\
\quad(n=524)\end{array}$ & $P$-value \\
\hline Age (years) & $43.4 \pm 7.7$ & $44.1 \pm 7.9$ & N.S. \\
\hline $\operatorname{AST}(\mathrm{U} / 1)^{\mathrm{a}}$ & $52 \pm 35$ & $55 \pm 39$ & N.S. \\
\hline $\operatorname{ALT}(\mathrm{U} / 1)^{\mathrm{a}}$ & $60 \pm 40$ & $63 \pm 47$ & N.S. \\
\hline $\begin{array}{l}\text { AST/ALT } \\
\text { (AAR) }\end{array}$ & $0.9 \pm 0.4$ & $1.0 \pm 0.5$ & N.S. \\
\hline $\operatorname{ALP}(\mathrm{U} / 1)^{\mathrm{a}}$ & $85 \pm 56$ & $87 \pm 55$ & N.S. \\
\hline Albumin $(\mathrm{g} / \mathrm{l})^{\mathrm{a}}$ & $40.2 \pm 4.1$ & $42.1 \pm 4.1$ & N.S. \\
\hline $\mathrm{INR}^{\mathrm{a}}$ & $1.2 \pm 0.3$ & $1.3 \pm 0.9$ & N.S. \\
\hline $\begin{array}{l}\text { Total bilirubin } \\
(\mathrm{mg} / \mathrm{dL})^{\mathrm{a}}\end{array}$ & $0.9 \pm 0.4$ & $0.8 \pm 0.3$ & N.S. \\
\hline $\begin{array}{l}\text { Platelet count } \\
\qquad\left(10^{9} / 1\right)^{\mathrm{a}}\end{array}$ & $179 \pm 54$ & $181 \pm 57$ & N.S. \\
\hline $\mathrm{APRI}^{\mathrm{b}}$ & $0.9 \pm 0.7$ & $1.0 \pm 0.9$ & N.S. \\
\hline $\operatorname{AFP}(\mathrm{U} / 1)^{\mathrm{a}}$ & $8.1 \pm 1$ & $10.2 \pm 1.0$ & N.S. \\
\hline $\begin{array}{l}\text { METAVIR } \\
\text { fibrosis stages }\end{array}$ & $\mathrm{N}(\%)$ & N (\%) & \\
\hline F0 & $26(8)$ & $41(7.8)$ & \\
\hline $\mathrm{F} 1$ & $73(22.5)$ & $121(23.1)$ & \\
\hline $\mathrm{F} 2$ & $73(22.5)$ & $116(22.1)$ & \\
\hline F3 & $75(23.1)$ & $119(22.7)$ & \\
\hline F4 & $77(23.8)$ & $127(24.2)$ & \\
\hline
\end{tabular}

Values were expressed as mean $\pm \mathrm{SD}$.

${ }^{a}$ Reference values: Aspartate aminotransferase (AST) (male up to 37 $\mathrm{U} / 1$, female up to $31 \mathrm{U} / 1$ ); alanine aminotransferase (ALT) (male up to $41 \mathrm{U} / 1$, female up to $31 \mathrm{U} / 1$ ); albumin $38-54 \mathrm{~g} / 1$; total bilirubin up to $1 \mathrm{mg} / \mathrm{dL}$; international normalized ratio (INR) 1; alkaline phosphatase (ALP) 22-92 U/1; platelet count $150-400 \times 10^{9} / 1$; alpha fetoprotein (AFP) up to 10 (U/1).

b Abbreviations: APRI: [(AST (U/1) / upper limits of normal)/platelet count $\left.\left(10^{9} / 1\right)\right] \times 100$.

${ }^{\mathrm{c}} P>0.05$ is considered nonsignificant; $P<0.05$ is considered significant; N.S., not significant.

a high significance on univariate analysis were entered in stepwise linear regression analysis to develop a model for identifying significant fibrosis. The diagnostic value of the developed score was assessed by calculating the area under the receiver-operating characteristic (ROC) curves. Diagnostic accuracy was calculated by sensitivity, specificity, positive predictive value (PPV), negative predictive value (NPV), and odds ratio. The cutoffs selected from the ROC curve were those that best identified significant fibrosis, advanced fibrosis, and cirrhosis.

\section{RESULTS}

\section{Patients Characteristics}

The score was constructed with data from 324 patients (the estimation study) and was then validated in 524 patients (the validation study). Patients characteristics at the time of liver biopsy are summarized in Table 1. There were no significant differences between the
TABLE 2. Variables Associated with the Presence of Significant Fibrosis (F2-F4) in the Estimation Group (324 Patients) by Univariate Analysis

\begin{tabular}{|c|c|c|c|}
\hline Marker & $\begin{array}{l}\text { Nonsignificant } \\
\text { fibrosis }(n=99)\end{array}$ & $\begin{array}{c}\text { Significant } \\
\text { fibrosis }(n=225)\end{array}$ & $P$-value ${ }^{\mathrm{c}}$ \\
\hline Age (years) & $38.3 \pm 7.9$ & $45.7 \pm 6.4$ & $<0.0001$ \\
\hline $\operatorname{AST}(\mathrm{U} / 1)^{\mathrm{a}}$ & $40 \pm 19$ & $58 \pm 39$ & $<0.0001$ \\
\hline $\operatorname{ALT}(\mathrm{U} / 1)^{\mathrm{a}}$ & $53 \pm 27$ & $63 \pm 45$ & 0.037 \\
\hline $\begin{array}{l}\text { AST/ ALT } \\
\text { (AAR) }\end{array}$ & $0.8 \pm 0.3$ & $1 \pm 0.4$ & $<0.0001$ \\
\hline $\operatorname{ALP}(\mathrm{U} / 1)^{\mathrm{a}}$ & $81 \pm 50$ & $90 \pm 58$ & 0.3 \\
\hline Albumin $(\mathrm{g} / \mathrm{l})^{\mathrm{a}}$ & $43.9 \pm 3.4$ & $41.4 \pm 4.2$ & $<0.0001$ \\
\hline $\mathrm{INR}^{\mathrm{a}}$ & $1.2 \pm 0.1$ & $1.2 \pm 0.3$ & 0.046 \\
\hline $\begin{array}{l}\text { Total bilirubin } \\
(\mathrm{mg} / \mathrm{dL})^{\mathrm{a}}\end{array}$ & $0.8 \pm 0.3$ & $0.9 \pm 0.4$ & 0.133 \\
\hline $\begin{array}{l}\text { Platelet count } \\
\qquad\left(10^{9} / 1\right)^{\mathrm{a}}\end{array}$ & $205 \pm 50$ & $168 \pm 53$ & $<0.0001$ \\
\hline $\mathrm{APRI}^{\mathrm{b}}$ & $0.6 \pm 0.3$ & $1.0 \pm 0.8$ & $<0.0001$ \\
\hline $\operatorname{AFP}(\mathrm{U} / 1)^{\mathrm{a}}$ & $2.7 \pm 0.1$ & $10.5 \pm 1.1$ & $<0.0001$ \\
\hline
\end{tabular}

Values were expressed as mean $\pm \mathrm{SD}$.

${ }^{a}$ Reference values: aspartate aminotransferase (AST) (male up to 37 $\mathrm{U} / 1$, female up to $31 \mathrm{U} / 1$ ); alanine aminotransferase (ALT) (male up to $41 \mathrm{U} / 1$, female up to $31 \mathrm{U} / 1$ ); albumin $38-54 \mathrm{~g} / 1$; total bilirubin up to $1 \mathrm{mg} / \mathrm{dL}$; international normalized ratio (INR) 1 ; alkaline phosphatase (ALP) 22-92 U/1; platelet count $150-400 \times 10^{9} / 1$; alpha fetoprotein (AFP) up to 10 (U/1).

b Abbreviations: APRI: [(AST (U/1) / upper limits of normal) / platelet count $\left.\left(10^{9} / 1\right)\right] \times 100$.

${ }^{\mathrm{c}} P>0.05$ is considered nonsignificant; $P<0.05$ is considered significant; $P<0.001$ is considered very significant and $P<0.0001$ is considered extremely significant.

estimation and the validation groups in any of the assessed variables or regarding the date of liver biopsy. In the estimation study, the patients were predominantly male, with a mean age of $42.8 \pm 8.1$ years at the time of biopsy. Overall, 225/324 (69.4\%) patients had significant fibrosis (F2-F4), 152/324 (46.9\%) had advanced fibrosis (F3-F4), and 77/324 (23.8\%) patients had cirrhosis (F4) (Table 1).

\section{Derivation of Biotechnology Research Center Score}

The main endpoint was the identification of patients with significant fibrosis vs. those without using a simple score based on routine markers. Univariate analysis of all variables tested in the estimation study revealed that age, AST, ALT, AAR, albumin, INR, platelet count, APRI, and AFP were significant $(P<0.05)$ and identified as predictors of fibrosis stage (Table 2). From these significant variables, only three variables (AFP, age, and platelet count) were identified as independent predictors in multivariate linear stepwise regression analysis. These markers were in a decreasing rank: AFP (AUC 0.79), age (AUC 0.76), and platelet count (AUC 0.72) as shown in 

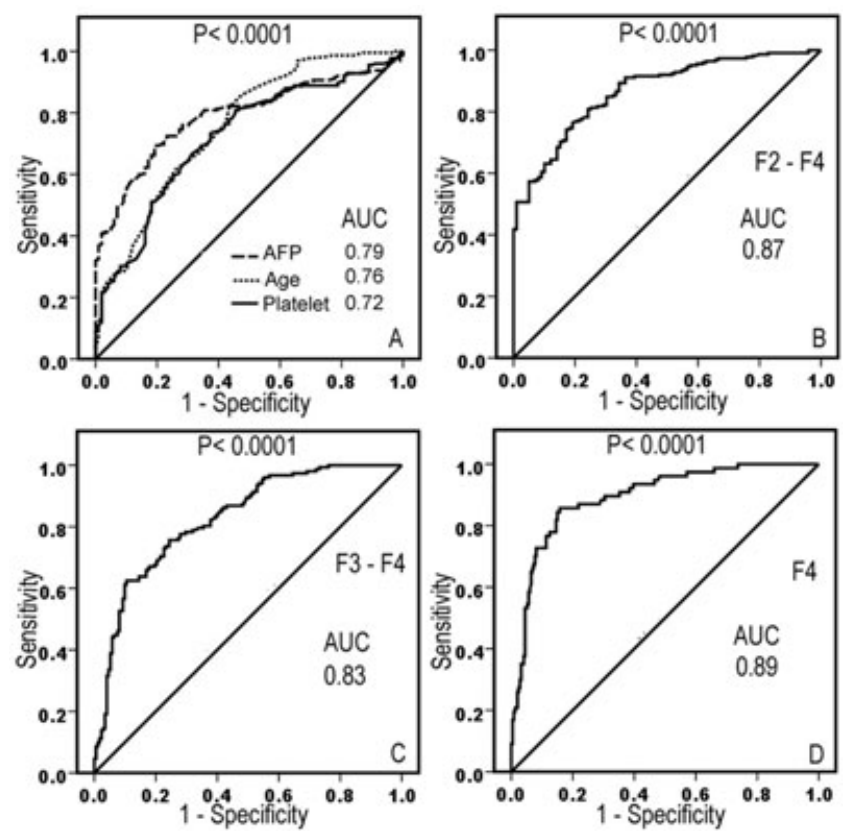

Fig. 1. Areas under receiver-operating characteristic curve (AUC) in chronic hepatitis $\mathrm{C}$ patients in the estimation study $(n=324)$ for (A) Candidate markers (AFP, age, and platelet count) for predicting significant fibrosis (F2-F4) (B) BRC score comprising AFP, age, and platelet count for predicting significant fibrosis (F2-F4) with an AUC of 0.87 (C) BRC score for predicting advanced fibrosis (F3-F4) with an AUC of 0.83 (D) BRC score for predicting cirrhosis (F4) with an AUC of 0.89 . Each point on the ROC plot represents a sensitivity/specificity pair corresponding to a particular decision threshold. An AUC of 1.0 is characteristic of an ideal test whereas an AUC of 0.5 or less indicates a test of no diagnostic value.

Figure 1A. Under the previous analysis, we constructed a Biotechnology Research Center (BRC) score combining AFP, age, and platelet count as a simple noninvasive score system:

$$
\begin{aligned}
& \text { BRC score }=1.02+0.4 \times \text { AFP }(\mathrm{U} / 1)+0.19 \\
& \times \text { Age }(\text { years })-0.02 \times \text { Platelet count }\left(10^{9} / 1\right)
\end{aligned}
$$

\section{Performance Characteristics of BRC Score}

The diagnostic value of this score was assessed in the estimation group by ROC curve showing an AUC of 0.87 for identifying significant fibrosis (Figure 1B). The BRC score was then applied on patients with advanced fibrosis and cirrhosis providing an AUC of 0.83 and 0.89 , respectively (Figure $1 \mathrm{C}$ and $\mathrm{D}$ ). The cutoffs were selected from the ROC curve and the diagnostic accuracy was calculated by sensitivity, specificity, PPV, NPV, and accuracy as shown in Table 3. For patients with BRC score greater than 7.2, 168 of $186(90 \%$ PPV) would have significant fibrosis and only 18 of 172 without significant fibrosis would be classi-
TABLE 3. Diagnostic Accuracy of BRC Score in the Estimation Study $(n=324)$

\begin{tabular}{lccc}
\hline & $\begin{array}{c}\text { Significant } \\
\text { fibrosis F2-F4 }\end{array}$ & $\begin{array}{c}\text { Advanced } \\
\text { fibrosis F3-F4 }\end{array}$ & Cirrhosis F4 \\
\hline Cutoff value & 7.2 & 8.2 & 9.2 \\
Sensitivity & 75 & 67 & 78 \\
Specificity & 82 & 80 & 86 \\
PPV $^{\text {a }}$ & 90 & 75 & 64 \\
NPV $^{\text {a }}$ & 59 & 85 & 96 \\
Accuracy & 77 & 74 & 84 \\
\hline
\end{tabular}

${ }^{\text {a }} \mathrm{PPV}$, positive predictive value; NPV, negative predictive value.
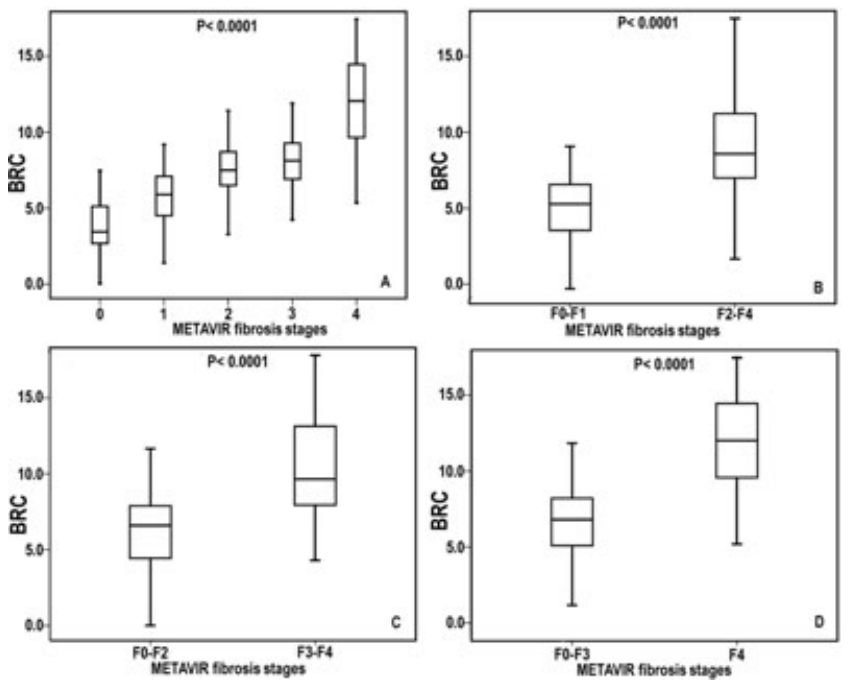

Fig. 2. Box plots for the BRC score in the estimation study $(n=324)$ in chronic hepatitis $\mathrm{C}$ patients (A) for each fibrosis stage; (B) for discriminating patients with significant fibrosis $(\mathrm{F} 2-\mathrm{F} 4)$ from those without (F0-F1); (C) for discriminating patients with advanced fibrosis (F3-F4) from those without (F0-F2) and (D) for discriminating patients with cirrhosis (F4) from those without (F0-F3). The box represents the interquartile range. The whiskers indicate the highest and lowest values and the line across the box indicates the median value. Overall significance of differences between the liver fibrosis groups was determined by $t$-test. $P>0.05$ is considered not significant, $P<0.05$ is considered significant, and $P<0.0001$ is considered extremely significant.

fied incorrectly. For patients with BRC score greater than $8.2,102$ of $136(75 \%$ PPV) would have advanced fibrosis and only 34 of 172 without advanced fibrosis would be identified falsely. At cutoff point 9.2, cirrhosis could be excluded with a superior NPV of $96 \%$ i.e., only $4 \%$ of patients with BRC score less than 9.2 had cirrhosis. For patients with BRC score greater than 9.2, 60 of $94(64 \%$ PPV) would have cirrhosis and only 34 of 247 without cirrhosis would be classified incorrectly. The BRC score distribution for patients in the respective fibrosis stage is depicted in Figure 2A. The median value for BRC score in $\mathrm{F} 0$ was $3.4, \mathrm{~F} 1$ was $5.9, \mathrm{~F} 2$ was $7.5, \mathrm{~F} 3$ was 8.1 , and F4 was 12.0. BRC score significantly correlated with liver 
TABLE 4. Performance of BRC Score in Discriminating Between Different Stages of Fibrosis in the Estimation Study $(n=324)$

\begin{tabular}{lccccc}
\hline METAVIR stage & $N^{\mathrm{a}}$ & AUC $^{\mathrm{a}}$ & $\mathrm{SE}^{\mathrm{a}}$ & $P$-value & $\left(95 \% \mathrm{CI}^{\mathrm{a}}\right)$ \\
\hline F0 vs. F1 & 26 vs. 73 & 0.80 & 0.05 & $<0.0001$ & $0.70-0.90$ \\
F1 vs. F2 & 73 vs. 73 & 0.73 & 0.04 & $<0.0001$ & $0.64-0.81$ \\
F2 vs. F3 & 73 vs. 75 & 0.57 & 0.05 & 0.130 & $0.48-0.67$ \\
F3 vs. F4 & 75 vs. 77 & 0.84 & 0.03 & $<0.0001$ & $0.78-0.91$
\end{tabular}

${ }^{a} N$, number of patients; AUC, area under the ROC curve; SE, standard error; CI, confidence interval.

${ }^{\mathrm{b}} P>0.05$ is considered nonsignificant, $P<0.05$ is considered significant, and $P<0.0001$ is considered extremely significant.

fibrosis stages (Spearman's rank correlation coefficient, $r=0.71, P<0.0001)$. The distribution of BRC score levels in relation to fibrosis stage is presented as box plots in Figure 2 (B-D). As shown in Figure 2(B-D), the differences were statistically significant in $\mathrm{F} 0-\mathrm{F} 1$ vs. F2-F4, F0-F2 vs. F3-F4, and F0-F3 vs. F4 $(P<0.0001)$. To eliminate any spectrum bias that may occur when there is a higher prevalence of any particular stage of fibrosis, ROC curve analysis was also performed comparing individual stages of METAVIR fibrosis i.e., F0 vs. F1, F1 vs. F2, F2 vs. F3, and finally F3 vs. F4 and the results are shown in Table 4.

\section{Validation Study}

In a second part of the study, we evaluated whether the predictive criteria identified in the estimation study were able to reproduce their predictive ability in a subsequent different, but related, group of patients. Considering the validation group, 362/524 (69.1\%) patients had significant fibrosis (F2-F4), 346/524 (47\%) had advanced fibrosis (F3-F4), and 127/524 (24.2\%) patients had cirrhosis (F4). The BRC score as well as other routinely available noninvasive predictors of fibrosis: AAR (17-19), APRI (20), Fibro- $\alpha$ score (21), and King's score (22) were applied to the 524 patients in the validation study to estimate its performance against that of these simple noninvasive tests. The correlation of each score to METAVIR fibrosis stage is depicted in Figure 3. ROC curves evaluating the diagnostic accuracies of these different noninvasive scores mentioned above in comparison with our developed score were constructed and superimposed to determine which score would have the most clinical utility to predict significant fibrosis (Fig. 4A). The AUC using the procedures described by Hanley and McNeil (25) was greatest for BRC score (0.85), then Fibro- $\alpha$ score (0.73), followed by King's score (0.70), APRI (0.68), AAR (0.59). In order to predict advanced fibrosis, ROC curves were also constructed and superimposed to determine which score would have the most clinical utility. The AUC was greatest for BRC score ( 0.82$)$, then Fibro- $\alpha$ score ( 0.74$)$, followed by King's

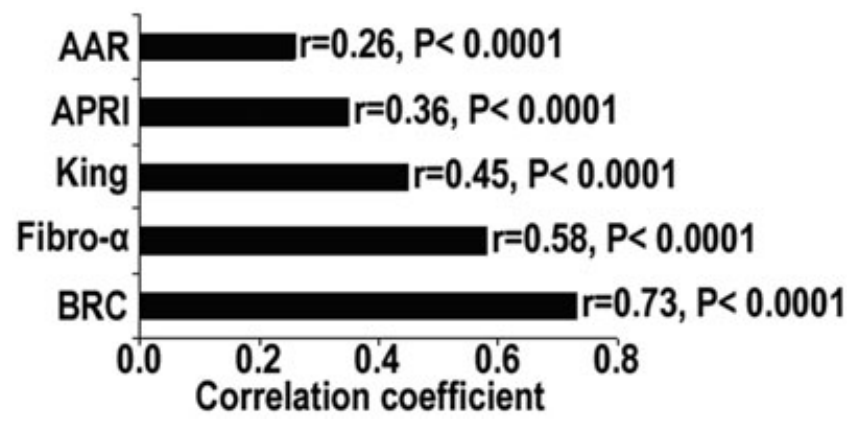

Fig. 3. Spearman's rank correlation of fibrosis stages (METAVIR fibrosis score) with simple noninvasive tests in the validation study $(n=524)$. $\mathrm{AAR}=\mathrm{AST} / \mathrm{ALT} ; \mathrm{APRI}=[(\mathrm{AST} /$ upper limit of normal $) /$ platelet count $(109 / 1)] \times 100 ;$ Fibro- $\alpha$ score $=1.35+$ AFP $(\mathrm{U} / 1) \times 0.009584+$ AAR $\times 0.243-$ platelet count $(109 / 1) \times 0.0016241$; King's score $=$ age $\times \operatorname{AST}(U / 1) \times$ INR / platelet count $(109 / 1)$; Biotechnology Research Center score $(\mathrm{BRC}$ score $)=1.02+0.4 \times$ AFP $(\mathrm{U} / \mathrm{l})+0.19 \times$ Age (years) $-0.02 \times$ Platelet count $(109 / 1)$.

score (0.70), APRI (0.68), AAR (0.58) (Fig. 4B). Likewise, to predict cirrhosis, the AUC was greatest for BRC score (0.88), then Fibro- $\alpha$ score (0.79), followed by King's score (0.71), APRI (0.68), AAR (0.59) as depicted in Figure 4C using the procedures described by Hanley and McNeil (25). Moreover, to compare our results with those of the previous reports, the sensitivity, specificity, PPV, NPV, and accuracy for these simple scores were calculated using categories and cutoff values exactly as originally described (Table 5). In addition, APRI and King's score originally focused on predicting the presence of significant fibrosis and cirrhosis whereas AAR focused on predicting cirrhosis. Herein, we selected the most balanced cutoff point for AAR, APRI, and King's score from the ROC analysis in order to predict categories not investigated in the original studies. It is evident that BRC score has better accuracy than AAR, APRI, Fibro- $\alpha$ score, and King's score in liver-fibrosis staging.

\section{DISCUSSION}

Nowadays, liver biopsy is considered the gold standard for the detection of fibrosis. Nevertheless, liver biopsy is an invasive procedure limiting both its acceptance by patients and their compliance for repeated procedures (5). For these reasons, several attempts have been made to find accurate noninvasive markers of disease activity and fibrosis. But some of these noninvasive means are expensive, need complicated calculations or involved tests that are generally not a part of the routine monitoring and investigations of patients with chronic liver disease. Laboratory variables used for discriminant scores must be more readily measurable, steady, and cheap to obtain. In light of the limited availability and high cost of many fibrosis markers, our results indicate that a regression model, 

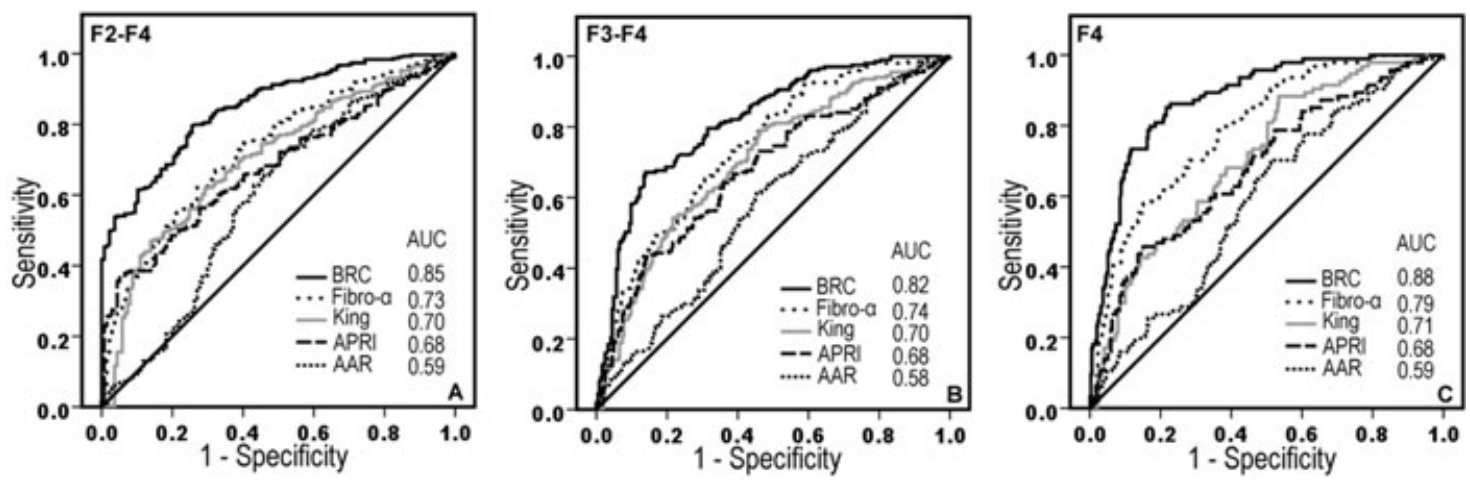

Fig. 4. Receiver operating characteristic curves of simple noninvasive tests evaluated for prediction of (A) significant fibrosis, (B) advanced fibrosis, (C) cirrhosis. AAR = aspartate aminotransferase (AST)/alanine aminotransferase (ALT); APRI = [(AST/ upper limit of normal) / platelet count $(109 / 1)] \times 100 ;$ Fibro- $\alpha$ score $=1.35+$ AFP $($ U/l $) \times 0.009584+$ AAR $\times 0.243-$ platelet count $(109 / 1) \times 0.0016241$; King's score $=$ age $\times$ AST $(\mathrm{U} / \mathrm{l}) \times \mathrm{INR} /$ platelet count $(109 / 1)$; Biotechnology Research Center score $($ BRC score $)=1.02+0.4 \times$ AFP $(\mathrm{U} / 1)+0.19 \times$ Age $($ years $)-0.02 \times$ Platelet count $(109 / 1)$.

derived from the combination of three simple noninvasive variables of fibrosis (AFP, age, and platelet count), has good diagnostic accuracy and reasonable predictive values for the detection of significant, advanced fibrosis and cirrhosis. On univariate and multivariate analyses, all variables except for ALP and total bilirubin were significant $(P<0.05)$ and identified as predictors of fibrosis stage. Multivariate regression modeling demonstrated that only age, platelet count, and AFP retained significance when combined with each other. The value of age as a marker of fibrosis seems obvious as fibrosis progression is timedependent (26-28) and several studies had identified age as independent predictor of fibrosis $(26,29-31)$. Hepatic fibrosis may lead to thrombocytopenia as a consequence of impaired synthesis of thrombopoietin and/or sequestering of platelets in an enlarged spleen (32). Many studies supported that platelet count alone may be clinically valuable as a noninvasive marker for liver fibrosis and cirrhosis $(33,34)$. Ono et al. $(35)$ reported the use of platelet count could discriminate F4 from F1-F3 in $75 \%-80 \%$ of patients with CHC. Platelet count has been combined with age in the age-platelet index (36) or with AST/ALT ratio and prothrombin time in the cirrhosis discriminant scores (37). In addition, platelet count is a component of APRI (20), Fibro- $\alpha$ (21), Forns index (26), model 3 (38), Fibrometer (39), and Fibroindex (40). In the present study, AFP was the most efficient marker among other markers with AUC of 0.79 for identifying patients with significant liver fibrosis. Thus, both platelet count and AFP level are easily measurable in daily practice for many $\mathrm{CHC}$ patients, and both meet the requirements described above. Under this analysis, BRC score (a combination of AFP, age together with platelet count) was established. Most of the noninvasive diagnosis methods have focused on predicting either the presence or absence of significant fibrosis and/or cirrhosis. For example, the index of Forns (26), which aims at predicting the absence of significant fibrosis (F0-F1) at a sensitivity of $94 \%$ and PPV of $40 \%$ (specificity of $51 \%$ and NPV of $96 \%$ ) at cutoff value $<4.2$; in patients with significant fibrosis (F2-F4) there is a low sensitivity of $30 \%$ and PPV of $66 \%$ at cutoff value $>6.9$. The index of Wai predicts significant fibrosis with a sensitivity of $41 \%$ and PPV of $88 \%$ (specificity $95 \%$ and NPV $64 \%$ ) at the cutoff value of 1.5 , and cirrhosis with a sensitivity of $57 \%$ and PPV of $57 \%$ (specificity $93 \%$ and NPV $93 \%$ ) at the cutoff value of 2.0 (20). Herein, BRC score could predict not only significant fibrosis but also advanced fibrosis and cirrhosis with a great accuracy. The diagnostic value of the BRC score was assessed by the ROC curve, showing an AUC of 0.87, 0.83 , and 0.89 for identifying significant fibrosis, advanced fibrosis, and cirrhosis, respectively in the estimation study. These results were reproduced in the validation study with no significant difference yielding AUC 0.85 for significant fibrosis, 0.82 for advanced fibrosis, and 0.88 for cirrhosis. The false negative and false positive results in this study can be explained in the light of studies performed by Pagliaro et al. and Poniachik et al. $(41,42)$. They showed that the sensitivity and specificity of liver biopsy were $68 \%$ and $99 \%$, respectively against laparoscopy. In the second part of this study, we aimed to evaluate the diagnostic accuracies of some noninvasive scores for assessing the degree of liver fibrosis in comparison with our developed score, BRC score. AAR (17-19), APRI (20), Fibro- $\alpha$ score (21), and King's score (22) were among these. Diagnostic accuracies for these different scores mentioned above were evaluated using categories and cutoff values exactly as originally described. Giannini et al, reported a high diagnostic accuracy of AAR $>1.16$ with $81.3 \%$ sensitivity and $55.3 \%$ specificity for the prediction of cirrhosis (16). However, AAR was not able to differentiate patients with liver cirrhosis from those without in our sample data. At value of $>1.0$, AAR has $40 \%$ sensitivity and $66 \%$ 
TABLE 5. Diagnostic Performances of BRC Score in the Validation Study $(n=524)$ and Comparison with AAR, APRI, Fibro- $\alpha$ Score, and King's Score and in Chronic Hepatitis C Patients

\begin{tabular}{|c|c|c|c|c|}
\hline \multirow[b]{2}{*}{ Score (reference) } & \multirow[b]{2}{*}{ Diagnostic performance $(\%)$} & \multicolumn{3}{|c|}{ METAVIR fibrosis stages } \\
\hline & & F2-F4 & F3-F4 & F4 \\
\hline \multirow[t]{7}{*}{$\mathrm{BRC}^{\mathrm{a}}$} & Cutoff & $>7.2$ & $>8.2$ & $>9.2$ \\
\hline & Sensitivity & 78 & 74 & 84 \\
\hline & Specificity & 78 & 76 & 80 \\
\hline & PPV & 89 & 74 & 57 \\
\hline & $\mathrm{NPV}^{\mathrm{a}}$ & 62 & 77 & 94 \\
\hline & Accuracy & 78 & 75 & 81 \\
\hline & Odd ratio $(95 \% \mathrm{CI})$ & $12.54(8.02-19.60)$ & $9.33(6.27-13.89)$ & $20.32(11.97-34.49)$ \\
\hline $\mathrm{AAR}^{\mathrm{a}}$ & Cutoff & $>0.8^{\mathrm{b}}$ & $>0.9^{\mathrm{b}}$ & $>1.0$ \\
\hline \multirow[t]{6}{*}{$(17-19)$} & Sensitivity & 67 & 54 & 40 \\
\hline & Specificity & 52 & 41 & 66 \\
\hline & $\mathrm{PPV}^{\mathrm{a}}$ & 76 & 45 & 27 \\
\hline & $\mathrm{NPV}^{\mathrm{a}}$ & 42 & 50 & 77 \\
\hline & Accuracy & 63 & 47 & 59 \\
\hline & Odd ratio $(95 \% \mathrm{CI})$ & $2.23(1.53-3.25)$ & $1.69(1.20-2.49)$ & $1.29(0.85-1.94)$ \\
\hline APRI $^{\mathrm{a}}$ & Cutoff & $>1.5$ & $>0.8 \S$ & $>2.0$ \\
\hline \multirow[t]{6}{*}{ (20) } & Sensitivity & 28 & 60 & 28 \\
\hline & Specificity & 98 & 67 & 93 \\
\hline & PPVf & 96 & 62 & 55 \\
\hline & NPVf & 38 & 66 & 80 \\
\hline & Accuracy & 50 & 64 & 77 \\
\hline & Odd ratio $(95 \% \mathrm{CI})$ & $15.34(5.54-42.49)$ & $3.14(2.19-4.49)$ & $4.88(2.84-8.40)$ \\
\hline Fibro-alpha & Cutoff & $>1.28$ & $>1.30$ & $>1.35$ \\
\hline \multirow[t]{6}{*}{ (21) } & Sensitivity & 78 & 83 & 82 \\
\hline & Specificity & 57 & 55 & 62 \\
\hline & $\mathrm{PPV}^{\mathrm{a}}$ & 80 & 62 & 41 \\
\hline & $\mathrm{NPV}^{\mathrm{a}}$ & 54 & 78 & 91 \\
\hline & Accuracy & 72 & 68 & 66 \\
\hline & Odd ratio $(95 \% \mathrm{CI})$ & $4.75(3.19-7.08)$ & $5.70(3.80-8.54)$ & $7.21(4.40-11.83)$ \\
\hline King & Cutoff & $\geq 12.3$ & $\geq 14^{\mathrm{b}}$ & $\geq 16.7$ \\
\hline \multirow[t]{6}{*}{$(22)$} & Sensitivity & 63 & 63 & 61 \\
\hline & Specificity & 68 & 65 & 65 \\
\hline & $\mathrm{PPV}^{\mathrm{a}}$ & 82 & 60 & 32 \\
\hline & $\mathrm{NPV}^{\mathrm{a}}$ & 45 & 68 & 86 \\
\hline & Accuracy & 65 & 64 & 64 \\
\hline & Odd ratio $(95 \% \mathrm{CI})$ & $3.67(2.39-5.62)$ & $3.23(2.19-4.77)$ & $2.87(1.80-4.58)$ \\
\hline
\end{tabular}

${ }^{\mathrm{a}}$ BRC, Biotechnology Research Center score $=1.02+0.4 \times$ AFP $(\mathrm{U} / \mathrm{l})+0.19 \times$ Age (years) $-0.02 \times$ Platelet count $\left(10^{9} / 1\right) ; \mathrm{AAR}, \mathrm{AST} / \mathrm{ALT}$; APRI, [(AST (U/1)/upper limits of normal)/platelet count $\left.\left(10^{9} / 1\right)\right] \times 100$; PPV, positive predictive value; NPV, negative predictive value. ${ }^{\mathrm{b}}$ Selected cutoff points in our study to predict categories not investigated in (17-20), and (22).

specificity for differentiating fibrosis from cirrhosis. This poor performance of AAR is similar to that reported by Lackner et al. (43). Consistent to our findings, Ahmad et al. (29) also reported low diagnostic accuracy of AAR in predicting cirrhosis (AUC 0.61). When cutoff values of 0.8 and 0.9 were selected from the ROC analysis for AAR so as to predict significant and advanced fibrosis, the results of our study found the diagnostic accuracy of AAR to be clearly low in identifying them with AUC of 0.59 and 0.58 , respectively. On the other hand, the lower AUC for APRI for predicting significant fibrosis and cirrhosis may be related to the differences in the study populations. In addition, differences in the reference ranges for AST may explain these discrepancies. Furthermore, we should notice that different staging systems using either a 5-stage (F0-F4) or a 7-stage (F0-F6) scale may also explain these discrepancies. When APRI was applied on advanced fibrosis, it generated diagnostic accuracy similar to that for significant fibrosis and cirrhosis in our sample data. The diagnostic accuracies of Fibro- $\alpha$ in identifying significant fibrosis, advanced fibrosis, and cirrhosis were $0.74,0.82$, and 0.80 , respectively in the original study (21) which was somewhat similar to those produced in our study. King's score was validated using cutoff values exactly as originally described. However, the accuracy of the original cutoff values was not found to be optimal and had AUCs lower than those reported previously in the original study (22). Cross et al. reported high diagnostic accuracy of the 
King's score for predicting cirrhosis (AUC 0.91) and significant fibrosis (AUC 0.79). At value of $\geq 16.7$, King's score has $86 \%$ sensitivity and $80 \%$ specificity for predicting cirrhosis whereas at value of $\geq 12.3$, King's score has $70 \%$ sensitivity and $85 \%$ specificity for predicting significant fibrosis. In contrast, our results found the diagnostic accuracy of King's score to be clearly inferior to that produced in the original study. Differences in the study populations and the histopathological assessment may explain these discrepancies. When King's score was applied on advanced fibrosis, it gave diagnostic accuracy similar to that for significant fibrosis and cirrhosis in our sample data. In conclusion, our findings showed that our newly developed score consisting of three readily available variables (AFP, age, and platelet count) is an accurate test for liver-fibrosis staging. It is evident that BRC score showed better performance than AAR, APRI, Fibro- $\alpha$ score, and King's score for liver-fibrosis staging in Egyptian CHC patients.

\section{REFERENCES}

1. Alter MJ, Kruszon-Moran D, Nainan OV, et al. The prevalence of hepatitis C virus infection in the United States, 1988 through 1994. N Engl J Med 1999;341:556-562.

2. WHO. Hepatitis C: Global prevalence. Wkly Epidemiol Rec 1997;72:341-344.

3. Frank C, Mohamed MK, Strickland GT, et al. The role of parenteral antischistosomal therapy in the spread of hepatitis $\mathrm{C}$ virus in Egypt. Lancet 2000;355:887-891.

4. Ray SC, Arthur RR, Carella A, Bukh J, Thomas DL. Genetic epidemiology of hepatitis C virus throughout Egypt. J Infect Dis 2000;182:698-707

5. Yeshua H, Oren R. Non invasive assessment of liver fibrosis. Ann Transplant 2008;13:5-11.

6. Marcellin P, Asselah T, Boyer N. Fibrosis and disease progression in hepatitis C. Hepatology 2002;36:S47-S56.

7. Fried MW, Shiffman ML, Reddy KR, et al. Peginterferon alfa-2a plus ribavirin for chronic hepatitis $\mathrm{C}$ virus infection. N Engl J Med 2002;347:975-982.

8. Lauer GM, Walker BD. Hepatitis C virus infection. N Engl J Med 2001;345:41-52.

9. Cadranel JF, Rufat P, Degos F. Practices of liver biopsy in France: Results of a prospective nationwide survey. For the Group of Epidemiology of the French Association for the Study of the Liver (AFEF). Hepatology 2000;32:477-481.

10. Bravo AA, Sheth SG, Chopra S. Liver biopsy. N Engl J Med 2001;344:495-500.

11. Bedossa $\mathrm{P}$, Dargère $\mathrm{D}$, Paradis V. Sampling variability of liver fibrosis in chronic hepatitis C. Hepatology 2003;38: 1449-1457.

12. Regev A, Berho M, Jeffers LJ, et al. Sampling error and intraobserver variation in liver biopsy in patients with chronic HCV infection. Am J Gastroenterol 2002;97:2614-2618.

13. Friedman SL. Liver fibrosis - from bench to bedside. J Hepatol 2003;38:S38-S53.

14. Sebastiani G, Alberti A. Non invasive fibrosis biomarkers reduce but not substitute the need for liver biopsy. World J Gastroenterol 2006;12:3682-3694.
15. Sebastiani G. Non-invasive assessment of liver fibrosis in chronic liver diseases: implementation in clinical practice and decisional algorithms. World J Gastroenterol 2009;15:2190-2203.

16. Denzer UW, Lüth S. Non-invasive diagnosis and monitoring of liver fibrosis and cirrhosis. Best Pract Res Clin Gastroenterol 2009;23:453-460.

17. Sheth SG, Flamm SL, Gordon FD, Chopra S. AST/ALT ratio predicts cirrhosis in patients with chronic hepatitis $\mathrm{C}$ virus infection. Am J Gastroenterol 1998;93:44 48.

18. Williams AL, Hoofnagle JH. Ratio of serum aspartate to alanine aminotransferase in chronic hepatitis. Relationship to cirrhosis. Gastroenterol 1988;95:734-739.

19. Giannini E, Risso D, Botta F, et al. Validity and clinical utility of the aspartate aminotransferase-alanine aminotransferase ratio in assessing disease severity and prognosis in patients with hepatitis $\mathrm{C}$ virus-related chronic liver disease. Arch Intern Med 2003;163:218224.

20. Wai CT, Greenson JK, Fontana RJ, et al. A simple noninvasive index can predict both significant fibrosis and cirrhosis in patients with chronic hepatitis C. Hepatology 2003;38:518-526.

21. Omran MM, Farid K, Emran TM, Attallah AA. Fibro- $\alpha$ score as a simple and useful non-invasive test for predicting significant liver fibrosis in chronic hepatitis C patients. Arab J Gastroenterol 2011;12:74-79.

22. Cross TJ, Rizzi P, Berry PA, Bruce M, Portmann B, Harrison PM. King's score: An accurate marker of cirrhosis in chronic hepatitis C. Eur J Gastroenterol Hepatol 2009;21:730-738.

23. Poynard T, Bedossa P, Opolon P. Natural history of liver fibrosis progression in patients with chronic hepatitis $\mathrm{C}$. The OBSVIRC, METAVIR, CLINIVIR, and DOSVIRC groups. Lancet 1997;349:825-832.

24. Manning DS, Afdhal NH. Diagnosis and quantitation of fibrosis. Gastroenterology 2008;134:1670-1681.

25. Hanley JA, McNeil BJ. A method of comparing the areas under receiver operating characteristic curves derived from the same cases. Radiology 1983;148:839-843.

26. Forns X, Ampurdanès S, Llovet JM, et al. Identification of chronic hepatitis $\mathrm{C}$ patients without hepatic fibrosis by a simple predictive model. Hepatology 2002;36:986-992.

27. Forns X, Ampurdanès S, Sanchez-Tapias JM, et al. Long-term follow-up of chronic hepatitis $\mathrm{C}$ in patients diagnosed at a tertiarycare center. J Hepatol 2001;35:265-271.

28. Poynard T, Ratziu V, Charlotte F, Goodman Z, McHutchison J, Albrecht $\mathrm{J}$. Rates and risk factors of liver fibrosis progression in patients with chronic hepatitis C. J Hepatol 2001;34:730-739.

29. Ahmad W, Ijaz B, Javed FT, et al. A comparison of four fibrosis indexes in chronic HCV: development of new fibrosis-cirrhosis index (FCI). BMC Gastroenterol 2011;11:44.

30. Vallet-Pichard A, Mallet V, Nalpas B, et al. FIB-4: An inexpensive and ccurate marker of fibrosis in HCV infection. comparison with liver biopsy and fibrotest. Hepatology 2007;46:32-36.

31. Hsieh YY, Tung SY, Lee IL, et al. FibroQ: An easy and useful noninvasive test for predicting liver fibrosis in patients with chronic viral hepatitis. Chang Gung Med J 2009;32:614-622.

32. Stauber RE, Lackner C. Noninvasive diagnosis of hepatic fibrosis in chronic hepatitis C. World J Gastroenterol 2007;13:4287-4294.

33. Myres RP, De Torres M, Imbert-Bismut F, Ratziu V, Charlotte F, Poynard T. MULTIVIRC group: Biochemical markers of fibrosis in patients with chronic hepatitis C: A comparison with prothrombin time, platelet count, and age-platelet index. Dig Dis Sci 2003;48:271-278.

34. Ghany MG, Lok AS, Everhart JE, et al. Predicting clinical and histologic outcomes based on standard laboratory tests in advanced chronic hepatitis C. Gasteroenterol 2010;138:136-146. 
35. Ono E, Shiratori Y, Okudaira T, et al. Platelet count reflects stage of chronic hepatitis C. Hepatol Res 1999;15:192-200.

36. Poynard T, Bedossa P. Age and platelet count: A simple index for predicting the presence of histological lesions in patients with antibodies to hepatitis $\mathrm{C}$ virus. METAVIR and CLINIVIR cooperative study groups. J Viral Hepatitis 1997;4: 199-208.

37. Bonacini M, Hadi G, Govindarajan S, Lindsay KL. Utility of a discriminant score for diagnosing advanced fibrosis or cirrhosis in patients with chronic hepatitis $\mathrm{C}$ virus infection. Am J Gastroenterol 1997;92:1302-1304.

38. Lok AS, Ghany MG, Goodman ZD, et al. Predicting cirrhosis in patients with hepatitis $\mathrm{C}$ based on standard laboratory tests: Results of the HALT-C cohort. Hepatology 2005;42:282-292.

39. Calès P, Oberti F, Michalak S, et al. A novel panel of blood markers to assess the degree of liver fibrosis. Hepatology 2005;42: 1373-1381.

40. Koda M, Matunaga Y, Kawakami M, Kishimoto Y, Suou T, Murawaki Y. FibroIndex, a practical index for predicting significant fibrosis in patients with chronic hepatitis C. Hepatology 2007;45:297306.

41. Pagliaro L, Rinaldi F, Craxì A, et al. Percutaneous blind biopsy versus laparoscopy with guided biopsy in diagnosis of cirrhosis. A prospective, randomized trial. Dig Dis Sci 1983;28:39-43.

42. Poniachik J, Bernstein DE, Reddy KR, et al. The role of laparoscopy in the diagnosis of cirrhosis. Gastrointest Endosc 1996;43:568-571.

43. Lackner C, Struber G, Liegl B, et al. Comparison and validation of simple noninvasive tests for prediction of fibrosis in chronic hepatitis C. Hepatology 2005;41:1376-1382. 